\title{
A study on FRAME model: Evidence from banking industry
}

\author{
Fazlollah Talebi ${ }^{\mathrm{a}}$ and Maryam Sasaniyan ${ }^{\mathrm{b}}$
}

${ }^{a}$ Institute of Applied Scientific Higher Education of Agriculture, Tehran, Iran

${ }^{b}$ Masters student in Management of Educational Planning, Kharazmi University, Tehran, Iran

\begin{tabular}{l}
\hline C H R O N I C L E \\
\hline Article history: \\
Received October 28, 2014 \\
Accepted 28 December 2014 \\
Available online \\
January 122015 \\
\hline Keywords: \\
FRAME \\
Bank Maskan \\
Mobile learning \\
Learner aspect
\end{tabular}

\section{Introduction}

During the past few years, three has been a growing concern on mobile learning. Many people believe mobile learning offers bigger access to necessary information, reduce cognitive load, and it can increase access to other information (Shneiderman \& Plaisant, 2005). Wireless, networked mobile devices may help shape sensitive learning experiences and facilitates to fit with the increasing amount of information in the world (Erstad, 2002; Cushion et al., 2003). The Framework for the Rational Analysis of Mobile Education (FRAME) model explains mobile learning features as a process in terms of mobile technologies, human learning capacities, and social interaction (Koole, 2009; Myers-Scotton, 2001). It describes various issues of information overload, knowledge navigation, and cooperation in learning. The framework gives insight for development of future mobile devices as well as learning materials, and an appropriate design of learning and teaching strategies for the purpose of mobile education. The FRAME model considers different technical characteristics of mobile devices along with social and

\footnotetext{
*Corresponding author.

E-mail addresses: maryam.sasaniyan@gmail.com (M. Sasaniyan)

(c) 2015 Growing Science Ltd. All rights reserved.

doi: 10.5267/j.msl.2015.1.003
} 
personal aspects of learning (Koole, 2009). This model is associated with concepts similar to those as detected in psychological theories. Nevertheless, the FRAME model also describes the role of technology beyond simply the effect of cultural or historic development. In this framework, the mobile device is considered as an active component to learning and social processes. The framework also places more emphasis on constructivism: the word rational refers to the "belief that reason is the primary source of knowledge and that reality is constructed rather than discovered" (Smith \& Ragan 1999, 15). Fig. 1 shows details of the FRAME model.

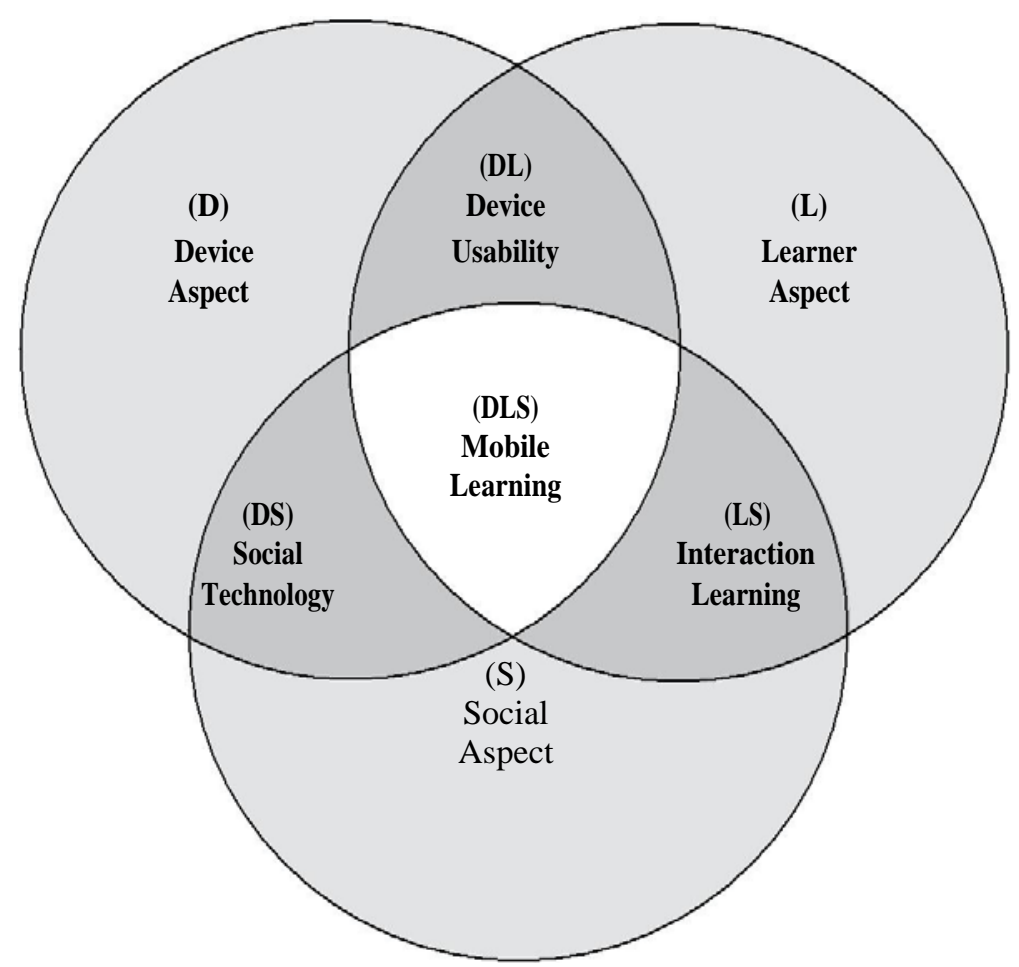

Fig. 1. The structure of FRAME model

In Fig. 1, the three circles indicate the device (D), learner (L), and social (S) characteristics. The intersections where two circles overlap include attributes, which is associated with both characteristics. Two attributes of the device usability (DL) and social technology (DS) intersections explain the affordances of mobile technology (Norman, 1999). The intersection, which is labelled as interaction learning (LS) includes instructional and learning theories. All three characteristics overlap at the primary intersection (DLS) in the center of the Venn diagram. The primary intersection, a convergence of all three characteristics, introduces an ideal mobile learning position. By evaluating the degree to which all the areas of the FRAME framework are utilized within a mobile learning circumstances, practitioners may apply the model to design more effective mobile learning experiences.

In FRAME model, the device aspect (D) is associated with the physical, technical, and functional characteristics of a mobile device. The physical characteristics incorporates input/ output capabilities to the machine such as power, processor speed, storage capabilities, compatibility, and expandability. These aspects result from the hardware and software design of the devices and have a substantial impact on the physical comfort levels of the users. It is essential to evaluate these characteristics since mobile learning devices give the interface between the mobile learner and the learning task(s) (Koole, 2009).

The learner aspect (L) considers an individual's cognitive abilities, memory, emotions, prior knowledge, and possible motivations. This aspect explains how learners implement what they already learn and how they encode, store, and transfer information (Koole, 2009). The social aspect considers the processes of social interaction and cooperation. Individuals have to follow the rules of cooperation 
to communicate, acquire necessary knowledge, and keep cultural practices. Rules of cooperation are detained by a learner's culture or the culture in which an interaction occur and in mobile learning, this culture could be physical or virtual (Koole, 2009). The device usability intersection includes elements that are associated with both the device (D) and learner (L) aspects. This section is associated with characteristics of mobile devices to cognitive tasks combined with the manipulation and storage of information. These processes, in turn, may influence on the user's sense of psychological comfort and satisfaction by influencing cognitive load, the capability to evaluate information, and the capability to physically move to various physical and virtual locations.

\section{The proposed study}

The proposed study of this paper implements the FRAME model in Iranian banking industry. There are three hypotheses as follows,

$\mathrm{H}_{1}$ : Alborz Province Housing Bank employees are ready to implement mobile learning in terms of device aspect.

$\mathrm{H}_{2}$ : Alborz Province Housing Bank employees are ready to implement mobile learning in terms of social aspect.

$\mathrm{H}_{3}$ : Alborz Province Housing Bank employees are ready to implement mobile learning in terms of learner aspect.

The study has been implemented among a sample of regular employees who worked for Bank Maskan in province of Alborz, Iran. The sample size is calculated as follows,

$$
n=\frac{N \times z_{\alpha / 2}^{2} \times p \times q}{\varepsilon^{2} \times(N-1)+z_{\alpha / 2}^{2} \times p \times q},
$$

where $N$ is the population size, $p=1-q$ represents the yes/no categories, $z_{\alpha / 2}$ is CDF of normal distribution and finally $\varepsilon$ is the error term. Since we have $p=0.5, z_{\alpha / 2}=1.96$ and $N=349$, the number of sample size is calculated as $n=152$. The questionnaire of this survey consists of 24 questions, where 4 questions are associated with general characteristics of the participants and 20 questions are related to three FRAME components; namely device (7 questions), social (6 questions) and learners (7 quesitions) and all questions are designed in Likert scale. To validate the questionnaire, we use content validity ratio (CVR) and verify the questionnaire using Cronbach alphas. For CVR test, we have asked 15 experts to judge about the questionnaire and for Cronbach alpha, we have distributed 30 questionnaires among some people who took part in our survey and measured Cronbach alpha. Table 1 demonstrates the results of CVR and Crobach numbers for the questionnaire.

\section{Table 1}

The summary of CVR and Cronbach alpha

\begin{tabular}{llcc}
\hline Item & Variable & CVR & Cronbach alpha \\
\hline 1 & Device & 0.59 & 0.70 \\
2 & Social & 0.62 & 0.79 \\
3 & Leaner & 0.54 & 0.78 \\
\hline
\end{tabular}

As we can observe from the results of Table 1, all components are within desirable levels and we can confirm the overall questionnaire. In our survey, $71.1 \%$ of the participants were male and $28.9 \%$ of them were female. Fig. 2 shows other personal characteristics of the participants. As we can observe from the results of Fig. 2, most people who took part in our survey were middle age with good university educations. In addition, they have maintained good job experiences since over $75 \%$ of them had at least 5 years of job experiences. 


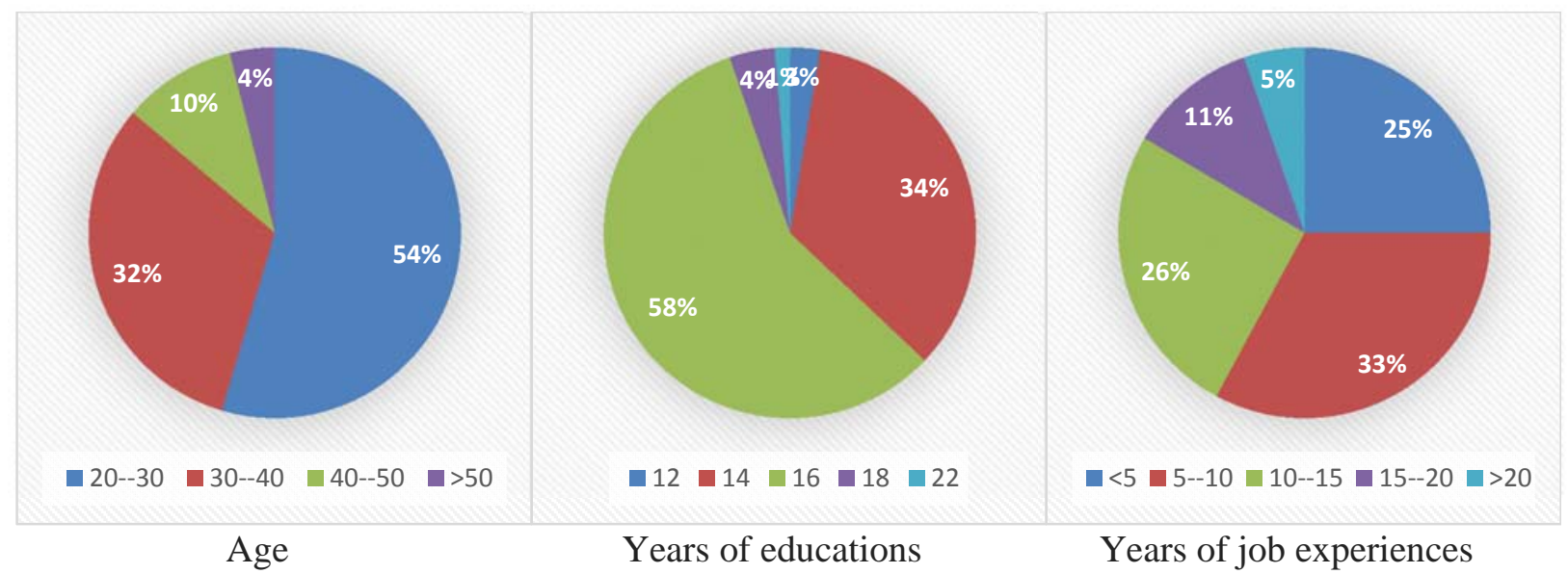

Fig. 2. Personal characteristics of the participants

The implementation of Kolmogorov-Smirnov has indicated that the date were normally distributed. Therefore, we use t-student to test the hypotheses of the survey.

\section{The results}

In this section, we present details of our findings on testing three hypotheses of the survey.

\subsection{Device effect}

The first hypothesis of the survey investigates whether the employees of Bank Maskan in province of Alborz are ready to adopt mobile learning in terms of device effect. Table 2 presents the summary of t-student test on this hypothesis.

\section{Table 2}

The summary of t-student test on examining the first hypothesis

\begin{tabular}{lcccccc}
\hline \multirow{2}{*}{ Hypothesis } & \multicolumn{7}{c}{ Measure $\mu<4$} \\
& t-student & Degree of freedom & Sig & Mean difference & Lower & Upper \\
\hline $\mathrm{H}_{1}$ & 16.739 & 151 & 0.000 & 0.82425 & 0.9215 & 0.727 \\
\hline
\end{tabular}

According to the results of Table 2, t-student value is significant when the level of significance is one percent. Therefore, the first hypothesis of the survey has been confirmed and we can confirm that the firm was ready to adopt mobile device learning.

\subsection{Social effect}

The second hypothesis of the survey tries to find out whether the employees of Bank Maskan in province of Alborz are ready to adopt mobile learning in terms of social effect. Table 3 shows the summary of t-student test on this hypothesis.

\section{Table 3}

The summary of t-student test on examining the second hypothesis

\begin{tabular}{lcccccc}
\hline \multirow{2}{*}{ Hypothesis } & \multicolumn{7}{c}{ Measure $\mu<4$} \\
& t-student & Degree of freedom & Sig & Mean difference & Lower & Upper \\
\hline $\mathrm{H}_{1}$ & 16.769 & 151 & 0.000 & 0.89803 & 1.0037 & 0.7924 \\
\hline
\end{tabular}


According to the results of Table 3, t-student value is significant when the level of significance is one percent. Therefore, the second hypothesis of the survey has been confirmed and we can confirm that the organization was ready to adopt mobile device learning, socially.

\subsection{Learner effect}

The third hypothesis of the survey tries to understand whether the employees of Bank Maskan in province of Alborz are ready to adopt mobile learning in terms of learner effect. Table 4 presents the summary of t-student test on this hypothesis.

\section{Table 4}

The summary of t-student test on examining the third hypothesis

\begin{tabular}{lcccccc}
\hline \multirow{2}{*}{ Hypothesis } & \multicolumn{7}{c}{ Measure $\mu<4$} \\
& t-student & Degree of freedom & Sig & Mean difference & Lower & Upper \\
\hline $\mathrm{H}_{1}$ & 16.481 & 151 & 0.000 & 0.82237 & 0.9210 & 0.7238 \\
\hline
\end{tabular}

According to the results of Table 4, t-student value is significant when the level of significance is one percent. Therefore, the third hypothesis of the survey has been confirmed and we can confirm that the organization was ready to adopt mobile device learning in terms of learner effect.

In summary, all three hypotheses of the survey have been confirmed and we can conclude that the case study of this survey was ready to adopt mobile device learning. Now, we are concerned to find the relative importance of each of three aspects using Freedman test. Table 5 demonstrates the results of our survey.

Table 5

The summary of Freedman test

\begin{tabular}{lcc}
\hline Rank & Aspect & Mean rank \\
\hline 1 & Learner & 2.11 \\
2 & Device & 1.97 \\
3 & Social & 1.92 \\
\hline Number of observations $=152$, Chi-Square $=3.296$, degree of freedom $=2$, Sig. $=0.000$
\end{tabular}

According to the results of Table 5, learner aspect is number one priority in our survey followed by device and social aspects.

\section{Conclusion}

The FRAME model may help practitioners and researchers leverage various benefits and to comprehend the complex nature of mobile learning, effectively. This paper has presented an empirical investigation to investigate whether mobile learning could be used in banking industry. The study has been accomplished in one of Iranian banks named Bank Maskan. Using t-student test, the study has determined that the firm was ready to adopt mobile learning in terms of device, social and learner aspects. In our survey, learner aspect is number one priority followed by device and social aspects.

\section{Acknowledgement}

The authors would like to thank the anonymous referees for constructive comments on earlier version of this paper. 


\section{References}

Ally, M. (Ed.). (2009). Mobile learning: Transforming the delivery of education and training. Athabasca University Press.

Cushion, C. J., Armour, K. M., \& Jones, R. L. (2003). Coach education and continuing professional development: Experience and learning to coach. Quest, 55(3), 215-230.

Erstad, O. (2002). Norwegian students using digital artifacts in project-based learning. Journal of Computer Assisted Learning, 18(4), 427-437.

Koole, M. L. (2009). A model for framing mobile learning. Mobile Learning: Transforming the Delivery of Education and Training, 1(2), 25-47.

Myers-Scotton, C. (2001). The matrix language frame model: Development and responses. Trends in Linguistics Studies and Monographs, 126, 23-58.

Norman, D. A. (1999). Affordance, conventions, and design. Interactions, 6(3), 38-43.

Shneiderman, S. B., \& Plaisant, C. (2005). Designing the user interface: Strategies for effective humancomputer interaction, $4^{\text {th }}$ ed., Pearson Addison Wesley, USA.

Smith, P., \& Ragan, T. (1999). Instructional design. $2^{\text {nd }}$ ed. Toronto: John Wiley \& Sons. 